\title{
Rule and optimization-based selection of car body parts for the application of tailor rolled blank technology
}

\author{
Niklas Klinke $^{1}$ (D) Vladimir Kobelev $^{2} \cdot$ Axel Schumacher $^{3}$
}

Received: 17 May 2021 / Revised: 21 September 2021 / Accepted: 14 October 2021 / Published online: 24 January 2022

(c) The Author(s) 2022

\begin{abstract}
The majority of parts in modern car bodies is manufactured from sheet metal. Rarely these parts are fully stressed due to design space restrictions and complex requirements. The usage of tailor rolled blanks (TRB) enables the reduction of sheet thickness in areas less loaded and thus reduces part weight. Technically most sheet metal parts are potentially suited for the application of TRB. Economic circumstances like the additional flexible rolling process and technology-specific nesting constraints limit the application to a subset of parts. The search for the best candidate parts taking mass and cost into account is currently challenging. This article presents an optimization strategy for the selection of the parts in a vehicle structure that are best suited for the application of TRB. As a first step, a priori preferencing is performed to select parts based on engineering rules. Using a reduced number of candidate parts, high quality metamodels are trained to perform multiobjective optimizations of all possible combinations of remaining parts, revealing the most efficient part selection under consideration of mass and cost.
\end{abstract}

Keywords Tailor rolled blanks $\cdot$ Part selection $\cdot$ Cost estimation $\cdot$ Multidisciplinary optimization

\section{Introduction}

Tailor rolled blanks (TRBs) are widely applied in automotive industry due their lightweight potential and the straightforward adaption to existing manufacturing processes, like deep-drawing and hot-stamping. Figure 1 shows the basic technology of TRB. Increased part cost due to flexible rolling process and additional scrap because of nesting constraints limit the application of TRB to a subset of parts in automotive bodies.

Automotive parts underlie a complex variety of partly conflicting goals and design constraints. Hence, their design

Responsible Eitor: Erdem Acar

Niklas Klinke

niklas.klinke@mubea.com

1 Mubea Tailor Rolled Blanks GmbH, New Body Development, P.O. Box 472, 57428 Attendorn, Germany

2 Mubea KG, Central Research and Development, P.O. Box 360, 57428 Attendorn, Germany

3 Chair for Optimization of Mechanical Structures, Faculty 7, University of Wuppertal, Gaußstraße 20, 42119 Wuppertal, Germany is challenging. With the increased design freedom of TRB, the complexity is further increased. This is the reason why optimization techniques have been used to design the thickness runs of Tailor Rolled Parts (TRPs) from the beginning on, as shown by Harzheim and Saul (2004).

Passenger vehicles have to withstand severe crash events, such as frontal or side impacts with other vehicles, trees, walls and other surroundings. In order to design body parts according to current legislative and customer crash test requirements, crash load cases are assessed virtually using finite element models.

Non-linearities like contacts, large deformations and material plasticity hinder the application of gradient-based optimization techniques whenever crash loads occur. Gradients would have to be calculated using finite difference, which is computationally demanding. Furthermore, it is highly unlikely to find a global optimum because of the noisy nature of crash events. Using metamodels trained from a design of experiments has proven to be a good option for the solution of crash problems as shown by Redhe et al. 


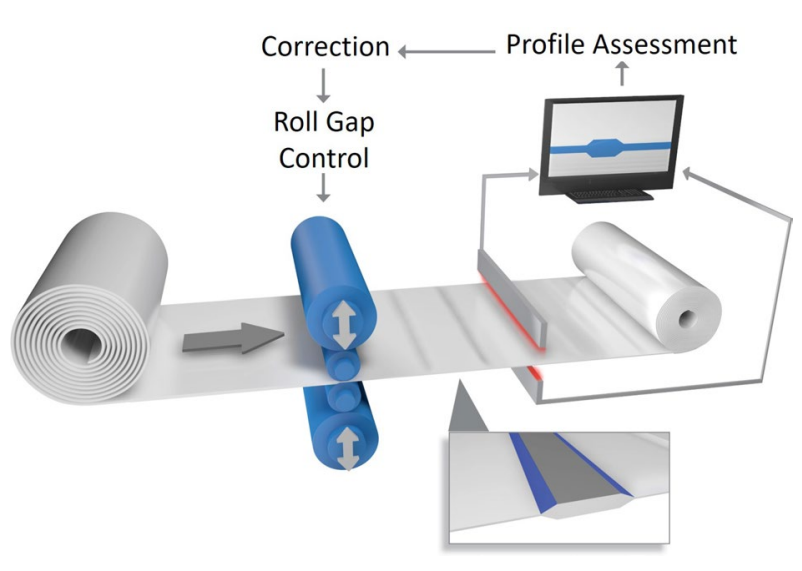

Fig. 1 Flexible rolling technology

(2002); Kodiyalam et al. (2004); Forsberg and Nilsson (2005). The application of metamodel-based optimization to the design of TRPs is presented by Chuang et al. (2008); Duan et al. (2015).

Most research work focuses on finding a minimum weight TRB design. Klinke and Schumacher (2021) present a multiobjective optimization scheme using a novel TRB-specific cost estimation, noting that in order to design a good TRB part often a goal conflict between minimum weight and minimum cost has to be solved. Determining which parts of the car body should be manufactured from TRB is a demanding task, because mass reduction has to be balanced with additional cost. Simply manufacturing all sheet metal parts of the car body out of TRB would be too costly, even if this would be the minimum weight design. Hence, the selection of the best subset of parts from a list of candidates is the task of this work.

The higher the number of design variables, the worse the approximation of the metamodels. The number of the design variables is therefore limited. For this reason the direct application of the multi-objective optimization scheme from Klinke and Schumacher (2021) with a corresponding high number of the design variables, as introduced by TRB, is not possible. A strategy has to be found that enables the systematic selection of parts well suited for the application of TRB under consideration of the economic constraints.

This paper presents a new strategy to select a subset of parts to a degree where optimization becomes possible, followed by a methodology that reveals the most promising combination of TRB and constant thickness parts under consideration of mass and cost goals. The potential of this strategy is shown, using the publicly available Toyota Yaris Crash model.

The work is structured as follows: After introducing a principal scheme for tailor rolled part selection in Sect. 2, Sect. 3 presents rules for selecting and prioritizing trb candidate parts. This scheme is applied to a finite element car body in order to select a subset of parts with which a metamodel-based optimization is computationally feasible. In Sect. 4 the optimization methodology as well as the parametrization and the metamodel training process are described. Afterwards the generated metamodel is used to perform a conventional single objective light weighting optimization with all candidate trb-parts. Since this approach is not goal leading when cost restrictions are given, a new approach is presented in Sect. 5. With the combinatoric multidisciplinary optimization every possible combination of trb- and constant thickness parts is optimized revealing the best compromise solutions of weight reduction and cost.

\section{Principal scheme for tailor rolled part selection}

The search of the most suitable TRB-parts aiming on reduction of mass $m$ and cost $p$ can be written as a multiobjective optimization task:

$Z_{f}=\min _{\mathbf{x}_{f}}\left[m\left(\mathbf{x}_{f}\right), p\left(\mathbf{x}_{f}\right)\right]$, with $\mathbf{x}_{f} \in \mathbb{R}^{n_{f}}$,

where $\mathbf{x}_{f}$ is the vector of design variables in case that every part would be a tailor rolled part. Thinking of around 100 parts in the BIW and around 5-10 design variables per part, it is comprehensible, that the task is hard to solve.

A strategy to decrease the number of possible solutions is the so-called a priori preferencing, presented by Hwang et al. (1979): An expert defines preferences in order to exclude potentially unsuitable solutions in a multi-objective decision problem. In our case this means, experts define preferences to exclude parts from the candidate list because it is known that those parts will not be in the desired solutions set. As a consequence this decreases the number of design variables $n_{s}$ :

$$
\begin{array}{r}
Z_{s}=\min _{\mathbf{x}_{s}}\left[m\left(\mathbf{x}_{s}\right), p\left(\mathbf{x}_{s}\right)\right], \\
\text { with } \mathbf{x}_{s} \in \mathbb{R}^{n_{s}} \\
\text { and } n_{s}<n_{f} .
\end{array}
$$

The assumption here is, that the solution set of the optimization $Z_{f}$ is comparable to $Z_{s}$.

For the formulation of preferences the following part characteristics play a role: blank width $w$, material grade $\sigma_{y}$, sheet thickness $t$ and scrap $s$. When the blank width exceeds the maximum coil width of the rolling mill, the manufacturing of this part from tailor rolled material is impossible. With a low material grade and a low sheet thickness, the load bearing capability of a part is low, 
hence the lightweight potential of such a part might be limited. Parts with a high scrap rate tend to be more costly than others, since the scrap material is also processed in the flexible rolling mill, but does not add value to the part.

Even if all part characteristics indicate the application of TRB, it is not guaranteed, that a thickness distribution is meaningful. As stated in the beginning, tailor rolled material can be applied effectively, when parts are not fully stressed, or in other words, inhomogeneously stressed. If this is not the case, TRB might not offer any weight saving, but increases cost. By analyzing the deviation of the load along an axis, the load inhomogeneity can be calculated, that quantifies how much the load is varying along a potential rolling direction of the part.

Using part characteristics as well as the load inhomogeneity the number of parts of interest and thus the number of design variables can be reduced based on an educated guess. The remaining parts can be optimized with the multidisciplinary optimization scheme from our previous work (Klinke and Schumacher 2021) to find a mass optimal design. Looking at cost and thickness ranges of each part one could simple select the parts offering a good compromise between mass and cost from this optimum. But since the car body is a complex system, whenever a subset of parts is selected for the application of TRB, the optimization has to be redone with this exact part choice. Otherwise, it is likely, that the thickness run of part A is not optimal anymore, when part $\mathrm{B}$ and part $\mathrm{C}$ are not anymore manufactured from TRB.

From this viewpoint, we propose to optimize every possible combination of tailor rolled and constant parts, that can be realized based on the design variables chosen after a priori preferencing. Afterwards the pareto optimal set of combinations can be isolated and the best compromise solutions offering minimal cost and weight are revealed. Based on the pareto optimal set, a posteriori preferencing is done: the project engineers decide on the final selection.

\section{Rule-based scheme for a priori preferencing}

In this work, we present a rule-based selection scheme that consists of two stages. The first stage evaluates part characteristics only, while the second one incorporates the actual loading of parts from a baseline design, evaluated in a set of load cases. Since it is common practice to design sheet metal structures based on constant thickness parts before investigating lightweight applications, it is possible that here the baseline design is coinciding with the optimal constant thickness design.

\subsection{A priori preferencing based on part characteristics}

Preferencing based on multiple characteristics can be done in several ways. For example, a scalar value can be calculated from the different characteristics, using a weighted average, forming a so-called decision criteria $\xi_{1}$ that expresses the suitability of TRB. Another way to find the most suited parts is to perform a multi-staged selection, where parts are excluded in each step due to their characteristics. We propose a mixture of both approaches, since certain criteria exist, that forbid the application of TRB, while others reveal tendencies.

Since the rolling mill can only process coils up to a certain maximum width $w_{\max }$, parts whose blank width $w$ exceeds this value are sorted out from the set $G_{0}$ of all parts $P_{i}$ :

$G_{0}=\left\{P_{i}\right\}$, where $i \in\left[1, \ldots, n_{p}\right]$,

$G_{1}=G_{0} \backslash\left\{P_{i} \mid w(i)>w_{\max }\right\}$.

Here $n_{p}$ is the number of parts in the initial set $G_{0}$. From the set of remaining parts $G_{1}$, only those are kept, that have a higher predefined thickness than $t_{\min }$, because otherwise thickness cannot be reduced much by TRB anymore:

$G_{2}=G_{1} \backslash\left\{P_{i} \mid t(i)<t_{\min }\right\}$.

To further decrease the number of parts, a decision criteria $\xi_{1}$ is determined based on sheet thickness $t$, scrap ratio $s$ and yield stress $\sigma_{y}$. The scrap ratio $s$ can be measured based on a nesting analysis performed with the part blank shape, after conducting an inverse forming simulation. The nesting algorithm places multiple blank shapes on a coil strip. The scrap rate can be measured as:

$s=1-\frac{m_{p}}{m_{r}}$,

where $m_{p}$ is the part mass and $m_{r}$ is the mass of the required raw material.

Prior to calculating $\xi_{1}$, all characteristics are normalized to a range between zero and one, such that parts with a high suitability are given the value one, while a low suitability is expressed as zero:

$$
\begin{aligned}
\tilde{t} & =\frac{t_{i}-t_{\min }}{t_{\max }-t_{\min }}, \\
\tilde{s} & =\frac{s_{\max }-s_{i}}{s_{\max }-s_{\min }}, \\
\tilde{\sigma_{y}} & =\frac{\sigma_{y, i}-\sigma_{y, \min }}{\sigma_{y, \max }-\sigma_{y, \min }}, \text { where } i \in\left[1, \ldots, n_{p}\right] .
\end{aligned}
$$

Normalization allows the calculation of the weighted average from characteristics of different orders of magnitude. 
The sheet thickness $t$ is used here again, since parts of high thickness have a greater potential for weight reduction. The scrap ratio $s$ is a measure for the part cost, while the yield stress $\sigma_{y}$ is an indicator for the level of load a part has to bear.

In this work, the decision criteria $\xi_{1}$ is calculated as the average of the normalized characteristics:

$\xi_{1}=\frac{\tilde{t}+\tilde{\sigma}_{y}+\tilde{s}}{3}$.

Depending on project goals, individual measures can also be of higher importance and a weighted average might be more suitable.

By setting the threshold for $\xi_{1}$ accordingly, the desired number of parts best suited for TRB can be selected:

$G_{3}=G_{2} \backslash\left\{P_{i} \mid \xi_{1}(i)<\xi_{\min }\right\}$.

\subsection{Extension of the criterion to include the analysis of the load inhomogeneity}

As stated in the introduction, tailored blanks are used in areas where sheet metal is inhomogeneously loaded. It seems likely, that parts with a less homogeneous loading are more suited for the application of TRB. Thus a methodology to analyze the load inhomogeneity is presented, which is then used for a slightly different decision criteria $\xi_{2}$.

A part is inhomogeneously loaded when the level of loading, expressed by a field response $\mathbf{v}$ like von-Mises stress $\sigma_{v m}$ or plastic strain $\varepsilon_{p l}$, is varying depending on the location inside the part. Since the local load level might be different over several load cases, the overall loading might be homogeneous. Hence, a combined loading has to be calculated first by determining the maximum load level of each field entity over all $n_{L}$ load cases. When the responses depend on time, also the maximum level over time $\tau$ has to be found:

$\mathbf{v}=\max _{\tau} \max _{i} \mathbf{v}_{i}(\tau)$, where $i \in\left[1,2, \ldots, n_{L}\right]$

Tailor rolled blanks have a thickness gradient in one direction only, thus the inhomogeneity $I$ along the desired rolling direction is of interest here. To determine $I$, the field response $\mathbf{v}$ is divided into $n_{b}$ segments along an desired axis. The segmentation is based on the coordinates of the blank shape. For every segment the mean of the field response is calculated: $\bar{v}_{j}$. The inhomogeneity can be expressed by calculating the standard deviation of the segment mean values to the mean of the part $\bar{v}$, which delivers a qualitative value within the same units:

$I(\mathbf{v})=\sqrt{\frac{1}{n_{b}} \sum_{j=1}^{n_{b}}\left(\bar{v}_{j}-\bar{v}\right)^{2}}$.
To further illustrate the calculation of inhomogeneity $I$, an example part is shown in Fig. 2. The stress distribution in blank coordinate system is shown in the background. The mean stress in each segment is shown as green bars, while the red line indicates the mean stress of the part.

Since the inhomogeneity gives a tendency rather than enabling the exclusion of parts, the normalized inhomogeneity $\tilde{I}(v)$ is used in addition to known characteristics in order to form the decision criteria $\xi_{2}$ which is used instead of $\xi_{1}$ to select the final part set $G_{3}$ :

$\tilde{I}=\frac{I_{i}-I_{\min }}{I_{\max }-I_{\min }}$, where $i \in\left[1, \ldots, n_{p}\right]$.

$\xi_{2}=\frac{\tilde{t}+\tilde{\sigma}_{y}+\tilde{s}+\tilde{I}}{4}$,

$G_{3}=G_{2} \backslash\left\{P_{i} \mid \xi_{2}(i)<\xi_{\min }\right\}$.

The threshold $\xi_{\min }$ can be chosen by the engineering team. Since it determines the number of parts in the subsequent optimization, it should be chosen based on engineering preference and computational budget.

\subsection{Application example: Toyota Yaris}

Modern vehicles have to withstand several crash events such as the side impact of a large and heavy vehicle (SUV or Pickup) or the roof loading in a roll over. These situations put a high load on the side structure of a vehicle. Due to design space restrictions and the geometry of doors and

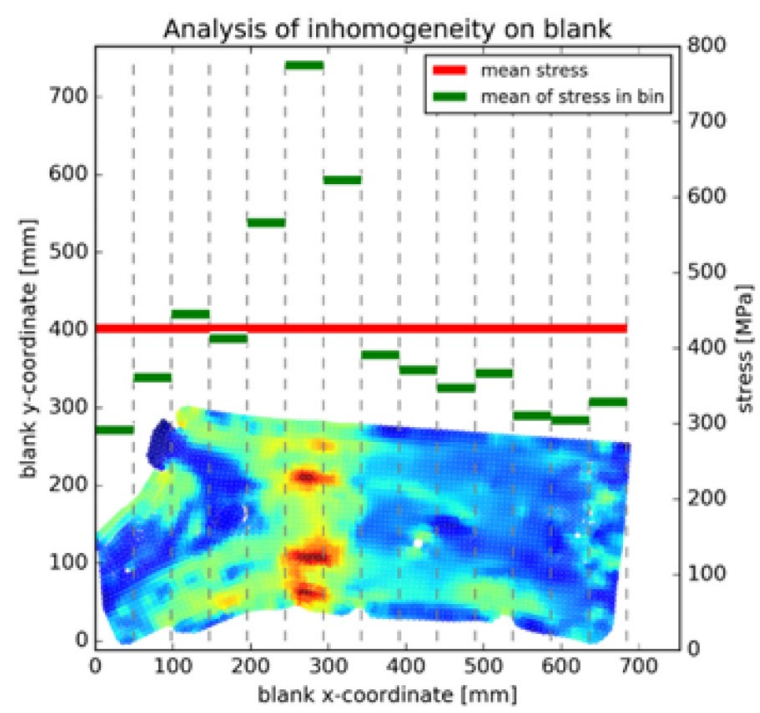

Fig. 2 Stress inhomogeneity of an example part 
crash barriers, the loading is not distributed homogeneously, leaving room for the application of TRB.

In order to reflect the effects of the side impact of a pickup, the Insurance Institute for Highway Safety (IIHS 2017) established a crash test, in which a deformable barrier with elevated impact height and a mass of $1500 \mathrm{~kg}$ hits the vehicle with $50 \mathrm{~km} / \mathrm{h}$. Lately the test protocol was adapted to new field data, using an altered barrier geometry with higher mass of $1900 \mathrm{~kg}$ and $60 \mathrm{~km} / \mathrm{h}$ impact speed (IIHS 2020). In addition to dummy measurements, both versions of the test protocol evaluate the structural performance based on the distance of the inner B-Pillar to the centerline of the driver seat, as shown in Fig. 3b. A good performance is reached when the inner B-Pillar stays more than $125 \mathrm{~mm}$ away from the driver seat centerline after the crash. Acceptable is a distance of 50-124 mm, while marginal describes a distance between $0 \mathrm{~mm}$ and $4.9 \mathrm{~mm}$. The passing of the centerline leads to a poor rating.

To demonstrate the application of the rule-based selection scheme a proper vehicle model is necessary. Here a publicly available finite element model of a Toyota Yaris from 2007 is used. The vehicle was reverse engineered and validated in several front crash load cases by the Center for Collision Safety and Analysis (CCSA 2016). In this process all parts were 3D-scanned and remodelled for FEM calculation. Material coupon tests were conducted to describe the material behavior. The model can be considered a standard in the crash simulation community. Its public availability and thus the good reproducibility of results were the reasons to choose this model even though it was not properly validated in IIHS side impact. Figure 3 a shows the simulation model for IIHS side impact simulation.

When evaluated according to the old test protocol, the structural performance of the finite element model turn out

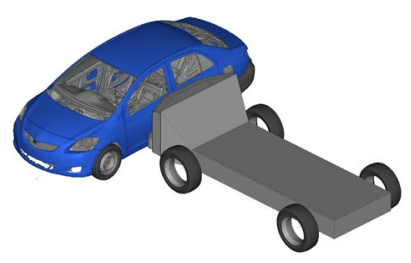

(a) Simulation model.

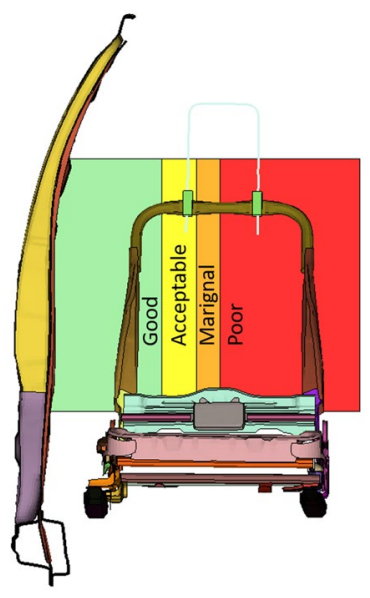

(b) Structural Rating.
Fig. 3 IIHS side impact to be poor. Hence we tuned the model with the goal to reach a good structural rating that enables a light weighing. At first the B-Pillar assembly, consisting of four parts, was simplified to a single sheet, as shown in Fig. 4a. Based on that model, the material of the six most sensitive parts in side crash was updated to hot-stamped 22MnB5. The thicknesses of those parts are updated by an optimization. The resulting structural rating can be reviewed in Fig. 9, while Table 1 shows a sheet thickness and performance summary of baseline (BM) and adapted model (AM).

When reviewing the side impact performance not every part of the body plays a role, hence 14 parts of the side frame were identified as potential TRB-parts based on engineering judgment (Fig. 5). Including all those parts in a metamodelbased optimization would increase the number of design variables drastically, which is why we limit ourselves to find the six most promising parts. This number has to be chosen based on project preferences.

Applying the presented rule-based selection scheme, the part characteristics as well as the decision criteria $\xi_{1}$,

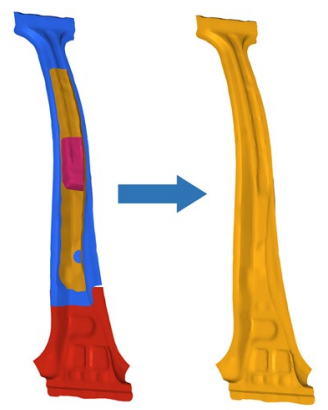

(a) Redesign of B-Pillar outer as a single sheet.

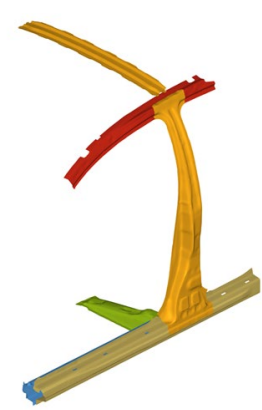

(b) Parts updated to hotstamped material.
Fig. 4 Yaris model updates

Table 1 Results of the optimization of the adapted model (AM) in comparison to the baseline model (BM)

\begin{tabular}{lll}
\hline & BM & AM \\
\hline$t_{\text {BPLR_OTR [mm] }}$ & 1.19 & 2.13 \\
$t_{\text {BPLR_OTR_LWR [mm] }}$ & 2.12 & - \\
$t_{\text {BPLR_REINF1 [mm] }}$ & 2.10 & - \\
$t_{\text {BPLR_REINF2 [mm] }}$ & 1.22 & - \\
$t_{\text {RKR_OTR [mm] }}$ & 1.43 & 1.47 \\
$t_{\text {RKR_INR [mm] }}$ [mm & 1.43 & 1.57 \\
$t_{\text {SQT2 }}[\mathrm{mm}]$ & 1.44 & 1.20 \\
$t_{\text {RBW }}[\mathrm{mm}]$ & 1.42 & 1.73 \\
$t_{\text {RR_OTR[mm] }}$ & 1.83 & 1.08 \\
Mass sum $\Sigma m[\mathrm{~kg}]$ & 32.61 & 31.65 \\
Seat Dist. $\delta_{\text {min }}[\mathrm{mm}]$ & -6.6 & 137.2 \\
IIHS Rating & Poor & Good \\
\hline
\end{tabular}




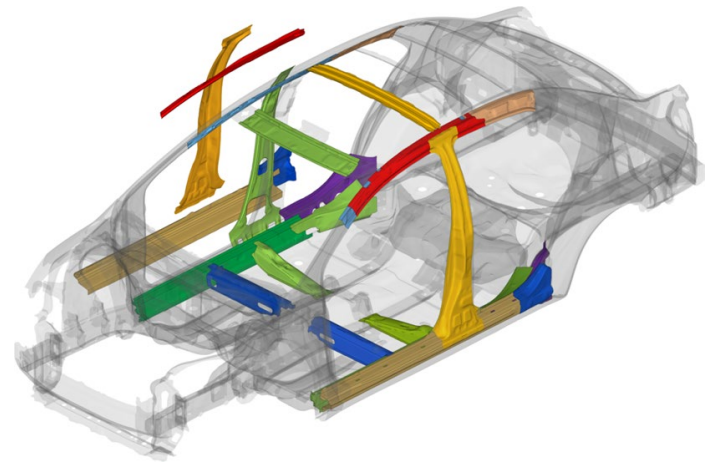

Fig. 5 Selected parts of the side structure

shown in Table 2 are calculated. It can be seen, that no part is excluded because of the blank width. The inner B-Pillar (BPLR_INR) and the front roof bow (RBW1) are sorted out, since their thickness $t$ is less than $1 \mathrm{~mm}$. To find the six most suited parts, the threshold for $\xi_{1}$ is set to be $\geq 45.15 \%$, which certainly reveals the same parts which were upgraded to hot-stamped material. The most suited part seems to be the middle roof bow (RBW2), followed by the outer rocker (RKR_OTR), while the outer B-Pillar (BPLR_OTR) is listed on position four.

As described before, the inhomogeneity can be calculated on arbitrary field responses. In order to derive a meaningful load inhomogeneity, a suitable field response has to be chosen. Possible responses are plastic strain $\varepsilon_{p l}$, von-Mises

Table 2 A priori selection based on part characteristics: blank width $w_{f}$, sheet thickness $t$, material yield $\sigma_{y}$ and scrap rate $s$. Additionally the decision criteria $\xi_{1}$ is shown

\begin{tabular}{llllll}
\hline & $\begin{array}{l}w_{f} \\
{[\mathrm{~mm}]}\end{array}$ & $\begin{array}{l}t \\
{[\mathrm{~mm}]}\end{array}$ & $\begin{array}{l}\sigma_{y} \\
{[\mathrm{MPa}]}\end{array}$ & $\begin{array}{l}s \\
{[\%]}\end{array}$ & $\begin{array}{l}\xi_{1} \\
{[\%]}\end{array}$ \\
\hline threshold & $\leq w_{\max }$ & $t \geq 1$ & & & $\geq 43.15$ \\
BPLR_INR & $\mathbf{3 9 3}$ & 0.99 & 270 & 56.96 & - \\
BPLR_OTR & $\mathbf{3 5 6}$ & $\mathbf{2 . 1 3}$ & 950 & 44.83 & $\mathbf{6 8 . 4 6}(4)$ \\
RBW1 & $\mathbf{1 8 2}$ & 0.97 & 270 & 15.22 & - \\
RBW2 & $\mathbf{1 2 1}$ & $\mathbf{1 . 7 3}$ & 950 & 9.04 & $\mathbf{8 5 . 9 2}(1)$ \\
RKR_INR & $\mathbf{2 2 7}$ & $\mathbf{1 . 4 7}$ & 950 & 6.39 & $\mathbf{8 0 . 3 6}(3)$ \\
RKR_OTR & $\mathbf{2 1 0}$ & $\mathbf{1 . 5 7}$ & 950 & 8.95 & $\mathbf{8 1 . 2 3}(2)$ \\
RKRE_INR & $\mathbf{3 8 4}$ & $\mathbf{1 . 9 0}$ & 270 & 40.14 & 31.95 \\
RKRE_OTR & $\mathbf{3 3 9}$ & $\mathbf{1 . 0 1}$ & 420 & 21.72 & 28.11 \\
FLRE_INR & $\mathbf{3 5 7}$ & $\mathbf{1 . 9 0}$ & 270 & 45.51 & 27.55 \\
RR_INR & $\mathbf{1 6 9}$ & $\mathbf{1 . 3 1}$ & 350 & 47.02 & 12.85 \\
RR_OTR & $\mathbf{1 5 5}$ & $\mathbf{1 . 0 8}$ & 950 & 37.59 & $\mathbf{4 3 . 1 5}(6)$ \\
RR2_INR & $\mathbf{1 4 1}$ & $\mathbf{1 . 0 7}$ & 350 & 21.39 & 26.70 \\
SQT1 & $\mathbf{2 8 8}$ & $\mathbf{1 . 4 6}$ & 350 & 19.81 & 39.55 \\
SQT2 & $\mathbf{1 8 7}$ & $\mathbf{1 . 2 0}$ & 950 & 20.59 & $\mathbf{6 0 . 6 7}(5)$ \\
\hline
\end{tabular}

Bold printed entries are above the threshold and thus part of the selection set stress $\sigma_{v m}$ and specific energy $u_{m}$. Where the specific energy $u_{m}$ is calculated from element mass $m$ and the strain energy $U$ and is thus based on strain energy density $u$ and element area $A$ :

$u=\frac{1}{2} \sigma \varepsilon, \quad U=u A, \quad u_{m}=\frac{U}{m}$.

Table 3 shows the inhomogeneity based on specific energy $I\left(u_{m}\right)$, plastic strain $I\left(\varepsilon_{p l}\right)$ and von-Mises stress $I\left(\sigma_{v m}\right)$. It can be concluded, that using the plastic strain or the stress response is not goal leading here, because parts of higher strength are preferred automatically and comparing parts with different materials would be questionable. Thus the energy-based inhomogeneity assessment $I\left(u_{m}\right)$ is our preferred choice and used to calculate $\xi_{2}$.

Compared to the results based on $\xi_{1}$, the selection stays the same, but the order of suitability changes. The loading of the outer B-Pillar (BPLR_OTR) is quite inhomogeneous, followed by the middle roof bow (RBW2) and the rocker sheets (RKR_INR and RKR_OTR).

The proposed inhomogeneity assessment is well suited to deliver another characteristic for a priori preferencing. Even if a simple strategy for the consideration of multiple load cases is presented here, load cases from different disciplines like stiffness or strength might have energy levels orders of magnitudes lower than crash. This would require an advanced calculation method for the combination of load levels in Eq. 9.

Table 3 inhomogeneity assessment based on different field responses: specific energy $I\left(u_{m}\right)$, plastic strain $I\left(\varepsilon_{p l}\right)$ and von-Mises stress $I\left(\sigma_{v m}\right)$. Additionally the decision criteria $\xi_{2}$ is shown

\begin{tabular}{lllll}
\hline & $\begin{array}{l}I\left(u_{m}\right) \\
{[\mathrm{J} / \mathrm{kg}]}\end{array}$ & $\begin{array}{l}I\left(\varepsilon_{p l}\right) \\
{[\%]}\end{array}$ & $\begin{array}{l}I\left(\sigma_{v m}\right) \\
{[\mathrm{MPa}]}\end{array}$ & $\begin{array}{l}\xi_{2} \\
{[\%]}\end{array}$ \\
\hline threshold & $\geq 335.75$ & $\geq 0.15$ & $\geq 33.78$ & $\geq 32.85$ \\
BPLR_INR & $\mathbf{8 6 4 . 4 4}(2)$ & $\mathbf{1 . 8 1}(1)$ & 28.94 & - \\
BPLR_OTR & $\mathbf{2 0 0 9 . 7 2}(1)$ & $\mathbf{0 . 3 5}(4)$ & $\mathbf{2 0 6 . 5 4}(1)$ & $\mathbf{7 6 . 3 5}(1)$ \\
RBW1 & 89.71 & 0.14 & 27.78 & - \\
RBW2 & 202.10 & 0.02 & $\mathbf{1 6 5 . 1 1}(2)$ & $\mathbf{6 6 . 4 0}(2)$ \\
RKR_INR & $\mathbf{5 1 8 . 0 6}(4)$ & $\mathbf{0 . 5 1}(3)$ & 30.94 & $\mathbf{6 6 . 2 6}(3)$ \\
RKR_OTR & $\mathbf{3 8 9 . 8 5}(5)$ & 0.10 & $\mathbf{9 1 . 1 3}(4)$ & $\mathbf{6 5 . 2 8}(4)$ \\
RKRE_INR & $\mathbf{3 3 5 . 7 5}(6)$ & $\mathbf{0 . 5 3}(2)$ & 13.42 & 27.63 \\
RKRE_OTR & 170.73 & $\mathbf{0 . 1 5}(6)$ & 2.89 & 22.64 \\
FLRE_INR & 71.17 & 0.06 & 4.36 & 20.95 \\
RR_INR & 119.05 & 0.13 & $\mathbf{3 9 . 1 1}(5)$ & 10.54 \\
RR_OTR & 86.44 & 0.03 & $\mathbf{1 3 8 . 0 7}(3)$ & $\mathbf{3 2 . 8 5}(6)$ \\
RR2_INR & 48.20 & 0.05 & 15.36 & 20.03 \\
SQT1 & 251.19 & $\mathbf{0 . 2 3}(5)$ & 13.82 & 32.25 \\
SQT2 & $\mathbf{6 1 9 . 9 4}(3)$ & 0.10 & $\mathbf{3 3 . 7 8}(6)$ & $\mathbf{5 2 . 7 9}(5)$ \\
\hline
\end{tabular}

Bold printed entries are above the threshold and thus part of the selection set 


\section{Selection based on optimization result}

Having determined the six most suited parts, we are now able to perform a TRB-optimization. Using the design evaluation and optimization strategy from our previous work (Klinke and Schumacher 2021; Klinke 2021), metamodels are trained sequentially until the prediction quality is not further improved.

This means that each iteration a batch of experiments is sampled using a space-filling approach with the software LS-Opt ${ }^{\circledR}$. Following the maximin-distance criterion it is ensured that new designs can be added subsequently without sacricing uniformity or equidistance of the design points as described by Stander et al. (2015).

Using the commercial software PSeven ${ }^{\circledR}$, metamodels for all responses are trained using different types of algorithms (Piecewise Linear Approximations, Polynomials, Gaussian Process Regression, ...) in parallel. The best model is selected based on Leave-One-Out cross validation (LOOCV) as described by Belyaev et al. (2016).

The optimization is then conducted on the metamodel and validated with the analysis model as shown in Fig. 6.

The underlying cae process estimates the effect of forming Tailor Rolled Blanks in a deep-drawing or a hot-stamping process. The resulting thickness distribution is mapped back to the crash analysis model. In addition the part's blank shape is utilized to conduct a TRB-nesting analysis. Based on a comprehensive cost model the piece price is estimated, as described by Klinke and Schumacher (2021); Klinke (2021).

In order to find optimal thickness configurations for TRBparts, the thickness run function has to be varied. The way the thickness run is parameterized influences the turnaround time of the optimization significantly, as shown by Klinke and Schumacher (2016). Focusing on reducing the computational effort, the most efficient way to parameterize the thickness distribution is a so-called Fixed Plateau Boundary (FPB) parametrization, where the positions of the areas of constant thickness (plateaus) are not moved.

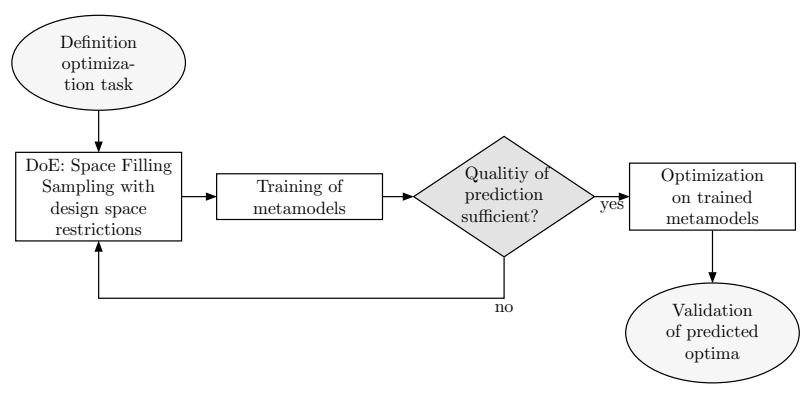

Fig. 6 Metamodel-based optimization scheme
The parts of interest are parameterized using an equally spaced fixed plateau boundary parametrization (es-FPB): the outer B-Pillar (BPLR_OTR) and the middle seat cross member (SQT2) are divided in $50 \mathrm{~mm}$ wide, the rocker sheets (RKR INR and RKR_OTR) as well as the outer roof rail (RR_OTR) and the middle roof bow (RBW2) in $100 \mathrm{~mm}$ wide plateaus and transitions. This leads to 43 design variables in total. The parametrization of the six parts is shown in Fig. 7a.

To assess side impact performance, metamodels are trained for the distances $\delta_{i}$ of several nodes on the inner B-Pillar to the middle of the driver seat, shown in Fig. $7 \mathrm{~b}$.

Following the optimization scheme showed in Fig. 6, an iterative metamodel training process is used. PSeven ${ }^{\circledR}$ is used for metamodel training. The model quality is evaluated in each step based on cross validation. The basic quality measures are calculated from the simulation responses $y_{i}$, the model prediction $\hat{y}_{i}$ as well as the mean response value $\bar{y}$ of $n_{d}$ design points:

$$
\begin{aligned}
e_{r m s} & =\sqrt{\frac{1}{n_{d}} \sum_{i=1}^{n_{d}}\left(y_{i}-\hat{y}_{i}\right)^{2}}, \\
e_{r r m s} & =\frac{e_{r m s}}{\sqrt{\frac{1}{n_{d}} \sum_{i=1}^{n_{d}}\left(y_{i}-\bar{y}\right)^{2}}}, \\
R^{2} & =1-\frac{\sum_{i=1}^{n_{d}}\left(y_{i}-\hat{y}_{i}\right)^{2}}{\sum_{i=1}^{n_{d}}\left(y_{i}-\bar{y}_{i}\right)^{2}}
\end{aligned}
$$

To derive single values that can be used to stop the iteration process, $e_{r m s, r r m s}$ and $R_{r m s}^{2}$ are derived from all $n_{r}$ responses:

$$
\begin{aligned}
e_{r m s, r r m s} & =\sqrt{\frac{1}{n_{r}} \sum_{i=1}^{n_{r}}\left(e_{r r m s, i}-\bar{e}_{r r m s}\right)^{2}}, \\
R_{r m s}^{2} & =\sqrt{\frac{1}{n_{r}} \sum_{i=1}^{n_{r}}\left(R_{i}^{2}-\bar{R}^{2}\right)^{2}} .
\end{aligned}
$$

Seven iterations, with 200 designs each were necessary to train metamodels until the quality indicators changed less

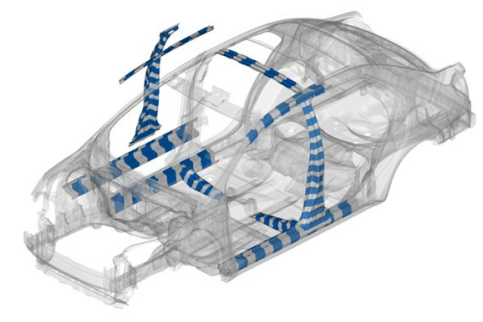

(a) TRB parametrization (gray: plateau, blue: ramp).

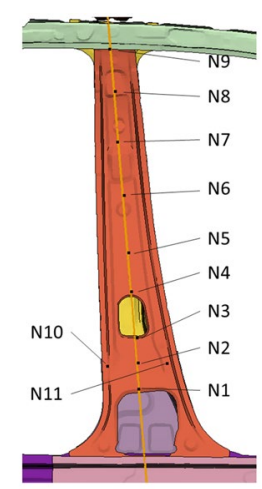

(b) Intrusion measurement nodes.

Fig. 7 Optimization parametrization and response definition for side impact 
the 5\%. The evolution of these measures can be reviewed in Fig. 8, while Table 4 shows the final values. Please note that theses measures where calculated based on cross validation scheme as explained by Belyaev et al. (2016). A detailed explanation of the used sequential metamodel training strategy is presented in Klinke and Schumacher (2021).

To find the lightest TRB design, the following optimization task is solved, based on the presented models, using the "Self-adaptive Differential Evolution" (SaDE) algorithm from the PyGMO python package of Biscani and Izzo (2020):

$$
\min _{x \in \mathbb{R}^{n}} m(x),
$$

where

$$
\begin{aligned}
& \delta(x) \geq 125 \mathrm{~mm}, \\
& g_{\text {TRB }}(x) \leq 0, \\
& 0.8 \mathrm{~mm} \leq x_{i} \leq 3.0 \mathrm{~mm} \quad i \in[1, \ldots, n] .
\end{aligned}
$$

Here $g_{T R B}$ describes manufacturing constraints, ensuring that a thickness profile can be rolled in the mill. The constraints, well-known from previous work of Harzheim and Saul (2004); Chuang et al. (2008); Klinke and Schumacher (2016, 2018), are shown here:

$g_{\mathrm{TRB}, 1}=1-\frac{\min _{i} t_{i}}{\max _{i} t_{i}}-r_{t, \max }$

$g_{\mathrm{TRB}, 2}=\frac{\left|t_{i}-t_{i+1}\right|}{50}-s_{\max }$

The optimal design is shown in Fig. 9a. Even if the minimum distance in side impact is just $120.9 \mathrm{~mm}$ and thus only an "Acceptable" rating, the deviation is in the range of expected metamodel accuracy.

It can be concluded from Fig. 9a, that five parts show significant thickness ranges, while the outer roof rail (RR_OTR) is down-gauged to the lower bound of $0.8 \mathrm{~mm}$. Since cost was not part of the optimization, this is the mass

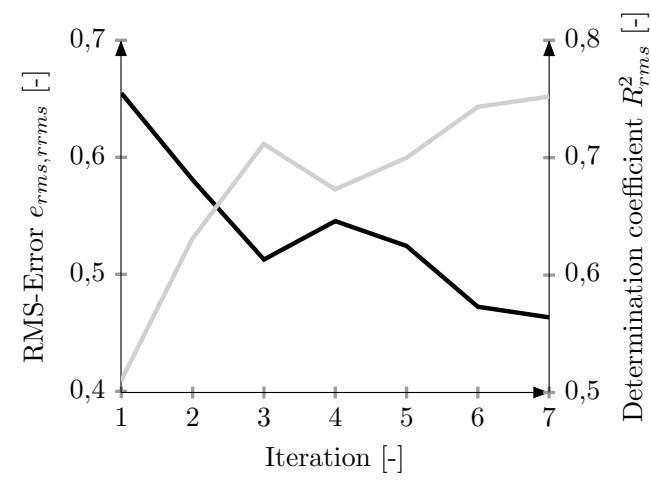

Fig. 8 Iterative model training for the IIHS side impact using six parts with 43 design variables: RMS value of the model quality measures ( $e_{\text {rms,rrms }}$ in black, $R_{r m s}^{2}$ in gray)
Table 4 Model quality of relevant responses

\begin{tabular}{llll}
\hline Response & $R^{2}[-]$ & $e_{\text {rms }}$ & $e_{\max }$ \\
\hline Mass $(\mathrm{RMS}) m[\mathrm{~kg}]$ & 1.000 & $2.90 \mathrm{e}-5$ & $5.60 \mathrm{e}-5$ \\
Distance $\delta_{N 1}[\mathrm{~mm}]$ & 0.949 & 5855 & 18,464 \\
Distance $\delta_{N 2}[\mathrm{~mm}]$ & 0.951 & 5305 & 16,342 \\
Distance $\delta_{N 3}[\mathrm{~mm}]$ & 0.902 & 7061 & 19,683 \\
Distance $\delta_{N 4}[\mathrm{~mm}]$ & 0.802 & 10,814 & 35,837 \\
Distance $\delta_{N 5}[\mathrm{~mm}]$ & 0.771 & 15,495 & 44,113 \\
Distance $\delta_{N 6}[\mathrm{~mm}]$ & 0.722 & 18,968 & 56,026 \\
Distance $\delta_{N 7}[\mathrm{~mm}]$ & 0.651 & 20,722 & 64,509 \\
Distance $\delta_{N 8}[\mathrm{~mm}]$ & 0.495 & 16,328 & 45,583 \\
Distance $\delta_{N 9}[\mathrm{~mm}]$ & 0.646 & 7,864 & 25,863 \\
Distance $\delta_{N 10}[\mathrm{~mm}]$ & 0.935 & 6416 & 18,830 \\
Distance $\delta_{N 11}[\mathrm{~mm}]$ & 0.943 & 5432 & 14,792 \\
Min. distance $\delta_{\min }[\mathrm{mm}]$ & 0.610 & 20,290 & 73,032 \\
\hline
\end{tabular}

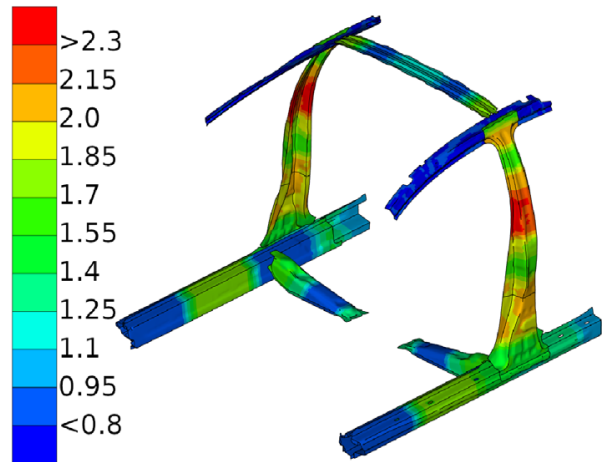

(a) Thickness distribution.

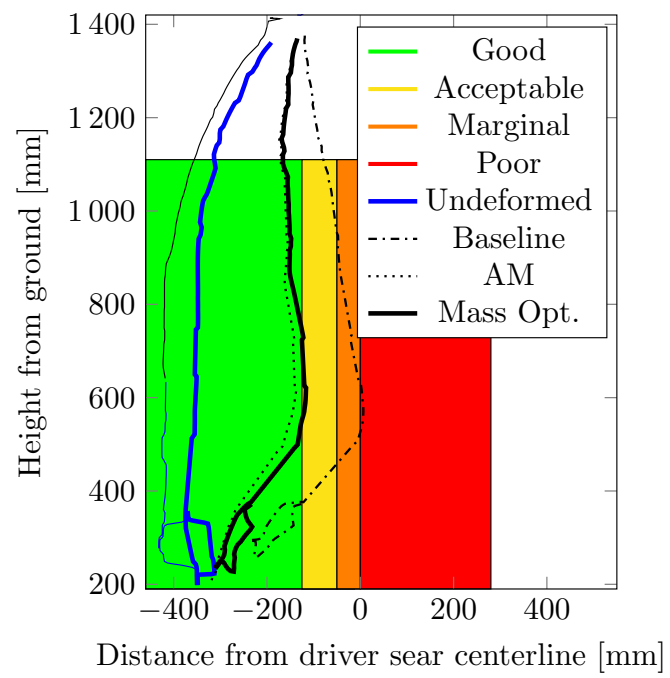

(b) IIHS side impact rating.

Fig. 9 Mass optimum (F) 
optimum, but no necessarily the most interesting economic solution.

Part price $p$ and lightweight cost can calculated for the mass optimum based on Klinke and Schumacher (2021); Klinke (2021), as shown in Table 5. Summing up weight and cost differences in the vehicle, the summed price $\Sigma p$ increases by $16.47 €$ while the summed weight $\Sigma m$ is decreasing by $4.576 \mathrm{~kg}$, which leads to lightweight cost of $3.6 € / \mathrm{kg}$.

In certain cases the lightweight cost of the mass optimum might be too high, necessitating further part selection. The trivial approach would be to just select the parts from Table 5, that offer a good compromise between cost and weight saving. Since the vehicle side frame is a complex system, it is not goal leading to simply pick a subset of parts, because the thickness run of the mass optimum is depending of the other parts and their thicknesses. Hence an optimization is necessary for every candidate selection, but since the choice for a particular selection can only be made knowing the optimum, every possible selection has to be optimized. Because no additional metamodel training is necessary, this task can be solved pretty fast.

\section{A posterior selection based on combinatoric multidisciplinary optimization}

Combinatoric multidisciplinary optimization means that every possible part selection, or manufacturing scenario is being optimized. A scenario is the combination of different manufacturing types per part (constant thickness or tailor rolled thickness). Thinking of a study with two possible TRB candidates, this leads to the scenarios shown in Table 6.

Using metamodel-based optimization enables the quick solving of several hundreds of such scenarios in a few hours. Using the cost model mentioned earlier, it is possible to include cost as a goal function in the optimization to find pareto optimal solutions of each manufacturing scenario.

Table 5 Analysis of the mass optimum: thickness range $\Delta t$ in each part, weight reduction $\Delta m$, price impact $\Delta p$ and lightweight cost (LWC) when compared to the adapted model

\begin{tabular}{lllll}
\hline Part & $\Delta t[\mathrm{~mm}]$ & $\Delta m[\mathrm{~kg}]$ & $\Delta p[€]$ & LWC [€/ kg] \\
\hline BPLR_OTR & 0.80 & 0.537 & 4.70 & 8.75 \\
RKR_OTR & 0.87 & 0.501 & 1.76 & 3.51 \\
RKR_INR & 0.88 & 0.784 & 1.00 & 1.28 \\
SQT2 & 0.76 & -0.024 & 1.33 & - \\
RBW & 0.89 & 0.486 & -0.17 & -0.35 \\
RR_OTR & 0.00 & 0.247 & -0.47 & -1.90 \\
\hline
\end{tabular}

Table 6 Possible scenarios in a study with two TRB candidates

\begin{tabular}{lll}
\hline Scenario & Part A & Part B \\
\hline 1 & Constant (c) & Constant (c) \\
2 & Constant (c) & TRB (t) \\
3 & TRB (t) & Constant (c) \\
4 & TRB (t) & TRB (t) \\
\hline
\end{tabular}

The trained metamodel offers the variation of six potential TRB-parts. For the outer B-Pillar in particular, different nesting scenarios apply. The more the B-Pillar shapes overlap each other on the coil, the lower the scrap rate. Unfortunately also the design freedom of the thickness run decreases resulting in less weight saving. In principle this is shown in Fig 10, the fully nested shapes in Fig 10a require the thickness run to by symmetric to the middle, while the thickness in the upper area of the B-Pillar can be reduced, when shapes are not nested (Fig 10b). Thus it is necessary to evaluate different nesting scenarios as shown in Klinke and Schumacher (2021); Klinke (2021).

Here the following nestings can be evaluated based on the presented parametrization: Fully overlapping nestingNT07, intermediate nestings-NT08, NT09, NT10, NT11, NT12 and no nesting-NN.

All other parts can be evaluated as part with constant thickness (const.) or as TRP, since no variation in the nesting is possible using the metamodel at hand. Combining these possibilities a total of 256 scenarios for the six parts have to be evaluated.

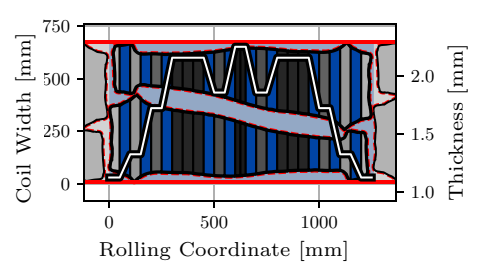

(a) NT07: Optimal thickness profile for overlapping nesting with symmetry at design variable T07.

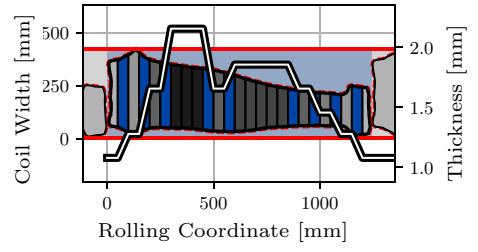

(b) NN: Optimal thickness profile for not nested blank without design variable symmetry.

Fig. 10 Influence of nesting on the optimal thickness run. (Blue: Transition zone, light gray to dark gray: Plateau thickness) 
Using the NSGA2 algorithm presented by Deb et al. (2002) from the PyGMO package, the following optimization task is solved for every scenario:

$$
\min _{x \in \mathbb{R}^{n}}[m(x), p(x)]
$$

where

$$
\begin{aligned}
& \delta(x) \geq 125 \mathrm{~mm}, \\
& g_{T R B}(x) \leq 0.0, \\
& 0.8 \mathrm{~mm} \leq x_{i} \leq 3.0 \mathrm{~mm} \quad i \in[1, \ldots, n] .
\end{aligned}
$$

Since the optimization tasks are independent of each other they can be solved in parallel on a HPC cluster in hours. In this case we used 96 individuals and 50000 generations.

Figure 11 shows the summed part cost over the summed mass of all optimal solutions for the six parts. Every point is an optimal solution of the particular scenario. Looking at the point markers the effect of the TRB application on each part can be understood. While the TRB application on the rocker sheets (RKR_OTR and RKR_INR) show a tendency towards the left, meaning decrease in weight with a fair cost increase, the outer B-Pillar (BPLR_OTR) tends to increase cost with decreasing amount of overlap in nesting.

To find the best alternatives among all solutions the pareto optimal set is isolated, as shown in Fig. 12. The shown points are validated using the analysis model, revealing slight deviations in side impact distance within the range of metamodel accuracy. Some characteristic points of the pareto front are marked and further analyzed in Table 7, Figs. 13 and 14. Please acknowledge, that also constant thickness parts show a thickness variation because of the integration of an automatic forming processes simulation.

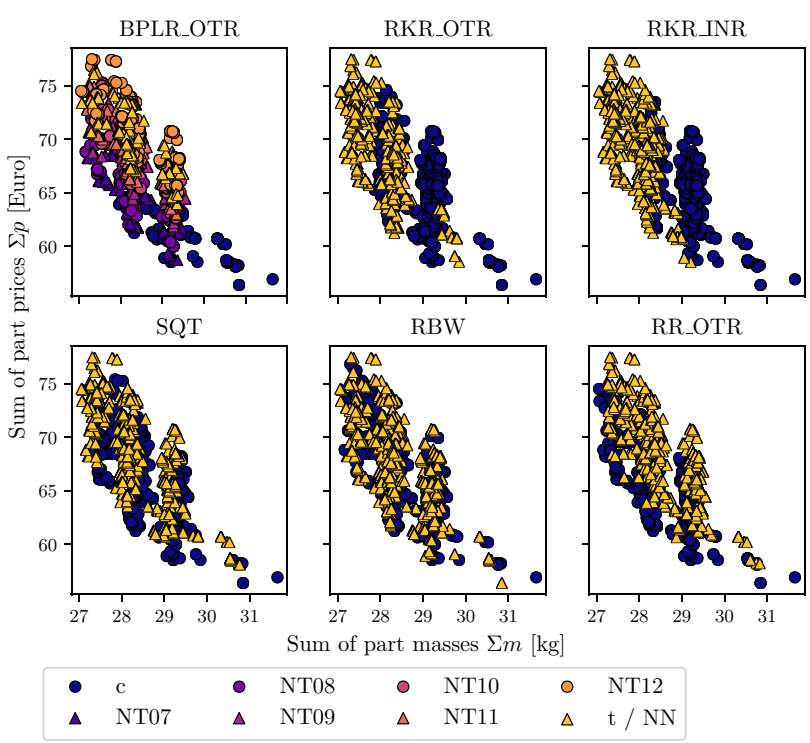

Fig. 11 Mass and price distribution of all possible scenarios

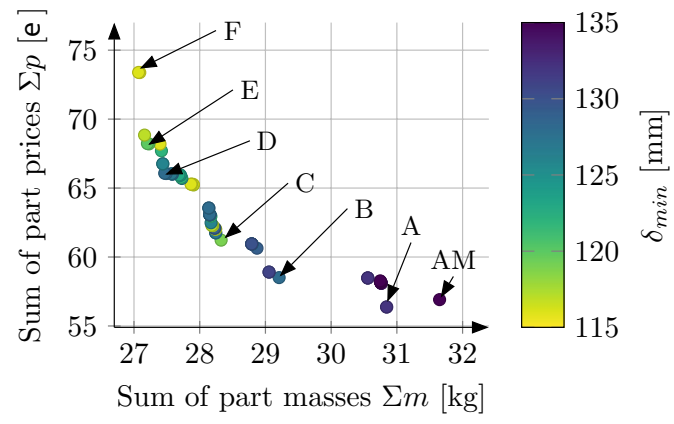

(a) Characteristic optima

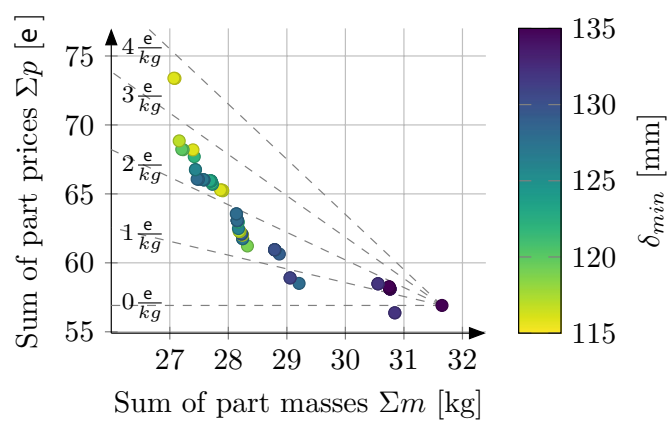

(b) Iso-lines with lightweight cost indication

Fig. 12 Pareto optimal solutions for the study part set. The coloring indicates the minimal distance to driver seat center line

Optimum A offers a weight reduction of $0.80 \mathrm{~kg}$, by increasing the TRB thickness of the outer ends of a roof bow, enabling a decrease in the rocker thicknesses and the outer roof rail. The summed cost of all parts ( 5 left, 5 right and roof bow) is slightly decreased by $0.54 €$, leading to lightweight cost of $-0.67 € / \mathrm{kg}$.

The weight reduction of Optimum B is $2.44 \mathrm{~kg}$, with a cost increase of $1.6 €$ and lightweight cost of $0.66 € / \mathrm{kg}$. In this design the outer rocker panel is made of TRB and enables a thickness decrease in the rocker inner.

When both rockers are made of TRB (Optimum C), $3.32 \mathrm{~kg}$ can be saved adding $4.31 €$ of cost, leading to $1.30 € / \mathrm{kg}$. It can be seen in Fig. 13 that the thickness run of the outer rocker changes quite significantly when the inner rocker has a thickness run.

Optimum D is the first optimum with a TRB B-Pillar. The weight reduction is $4.18 \mathrm{~kg}$ with additional cost of $9.16 €$ and lightweight cost of $2.19 € / \mathrm{kg}$. In this scenario the B-Pillar is nested symmetrically and fully overlapping.

Manufacturing the seat cross member (SQT2) of TRB, as done in Optimum E, adds an extra of $0.27 \mathrm{~kg}$ to the weight reduction, leading to $4.45 \mathrm{~kg}$ of total weight reduction compared to the adapted model. Costing an extra of $11.32 €$, the lightweight cost is $2.54 € / \mathrm{kg}$. 
BPLR_OTR

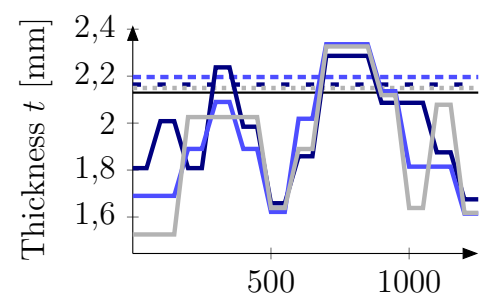

Rolling coordinate $c[\mathrm{~mm}]$ SQT2

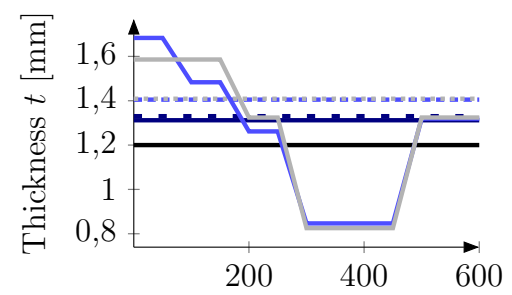

Rolling coordinate $c[\mathrm{~mm}]$
RKR_OTR

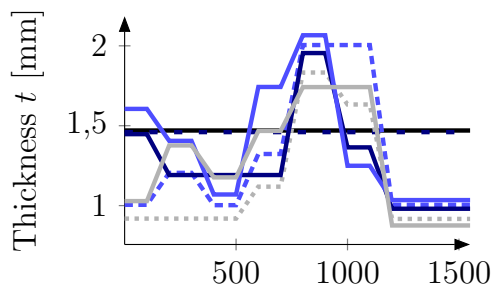

Rolling coordinate $c[\mathrm{~mm}]$

RBW2

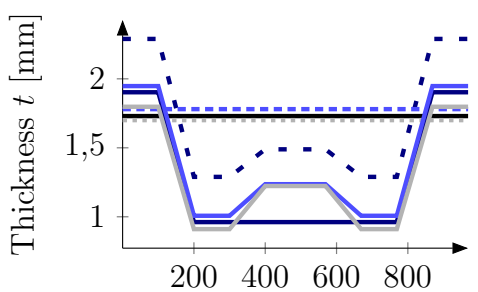

Rolling coordinate $c[\mathrm{~mm}]$
RKR_INR

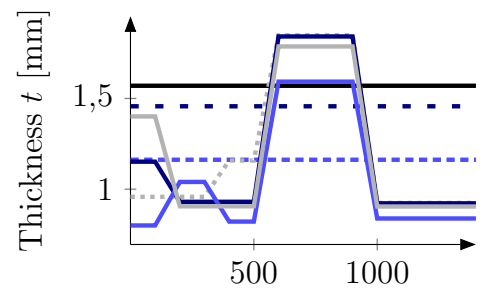

Rolling coordinate $c[\mathrm{~mm}]$

RR_OTR

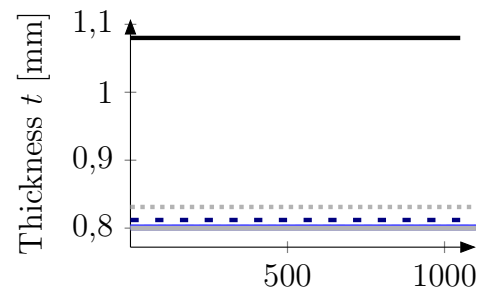

Rolling coordinate $c[\mathrm{~mm}]$

$$
\text { - } \mathrm{AM}=\mathrm{A} \ldots \mathrm{B} \ldots \mathrm{C}-\mathrm{D}-\mathrm{E}-\mathrm{F}
$$

Fig. 13 Thickness runs of the selected characteristic optima

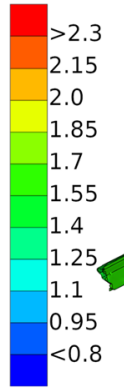

(a) Adapted model (AM).

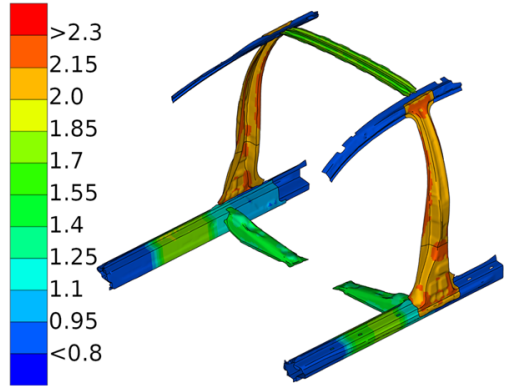

(d) Optimum C.

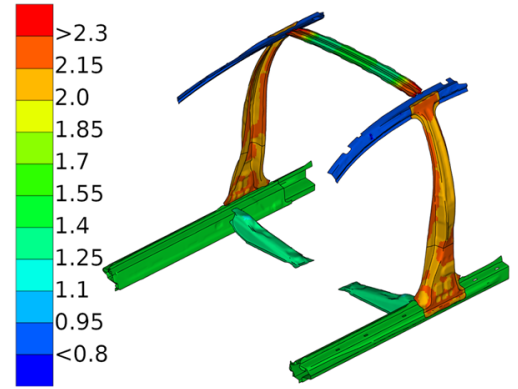

(b) Optimum A.
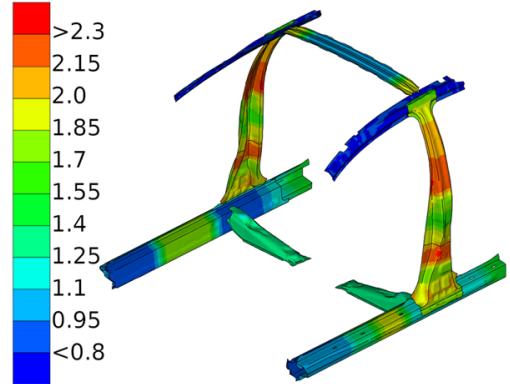

(e) Optimum D.

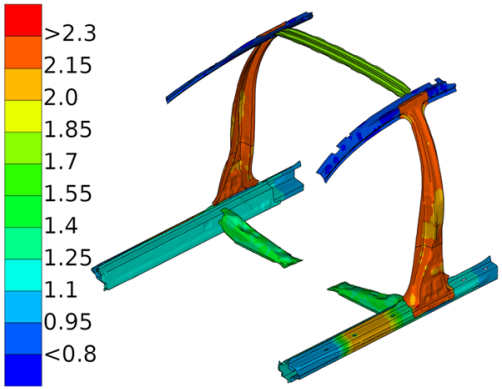

(c) Optimum B.
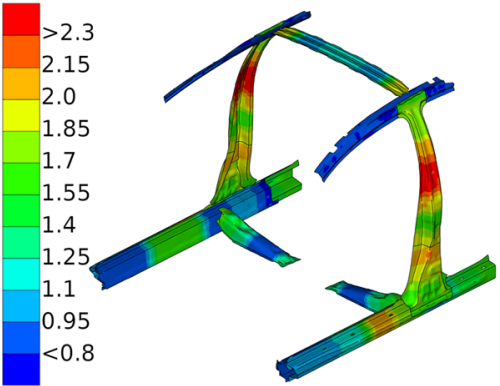

(f) Optimum E.

Fig. 14 Thickness distributions of the selection characteristic points of the pareto frontier 
Table 7 Result summary for the selected characteristic optima

\begin{tabular}{|c|c|c|c|c|c|c|c|}
\hline & $\mathrm{AM}$ & A & B & $\mathrm{C}$ & $\mathrm{D}$ & $\mathrm{E}$ & $\mathrm{F}$ \\
\hline \multicolumn{8}{|l|}{ Manufacturing scenario } \\
\hline BPLR_OTR & const. & const. & const. & const. & NT07 & NT07 & NN \\
\hline RKR_OTR & const. & const. & TRP & TRP & TRP & TRP & TRP \\
\hline RKR_INR & const. & const. & const. & TRP & TRP & TRP & TRP \\
\hline SQT2 & const. & const. & const. & const. & const. & TRP & TRP \\
\hline RBW & const. & TRP & const. & const. & TRP & TRP & TRP \\
\hline RR_OTR & const. & const. & const. & const. & const. & const. & const. \\
\hline \multicolumn{8}{|l|}{ Thicknesses } \\
\hline $\mathbf{t}_{\mathrm{BPLR}-} \mathrm{OTR}[\mathrm{mm}]$ & 2.13 & 2.16 & 2.20 & 2.15 & $1.67-2.29$ & $1.61-2.34$ & $1.53-2.33$ \\
\hline $\mathbf{t}_{\mathrm{RKR}-\mathrm{OTR} \text { [mm] }}$ & 1.47 & 1.46 & $1.00-2.00$ & $0.92-1.83$ & $0.98-1.95$ & $1.03-2.06$ & $0.86-1.74$ \\
\hline $\mathbf{t}_{\mathrm{RKR} \_}$INR [mm] & 1.57 & 1.46 & 1.16 & $0.92-1.84$ & $0.92-1.84$ & $0.80-1.59$ & $0.90-1.79$ \\
\hline $\mathbf{t}_{\mathrm{SQT} 2}[\mathrm{~mm}]$ & 1.20 & 1.33 & 1.41 & 1.41 & 1.31 & $0.85-1.68$ & $0.83-1.59$ \\
\hline $\mathbf{t}_{\mathrm{RBW}}[\mathrm{mm}]$ & 1.73 & $1.29-2.29$ & 1.78 & 1.70 & $0.96-1.90$ & $1.00-1.95$ & $0.91-180$ \\
\hline $\mathbf{t}_{\mathrm{RR}-O T R}[\mathrm{~mm}]$ & 1.08 & 0.81 & 0.80 & 0.83 & 0.80 & 0.80 & 0.80 \\
\hline \multicolumn{8}{|l|}{ Results } \\
\hline$m_{\text {BPLR_OTR }[\mathrm{kg}]}$ & 9.87 & 10.04 & 10.19 & 9.97 & 9.15 & 8.86 & 8.80 \\
\hline$m_{\text {RKR_OTR }[\mathrm{kg}]}$ & 8.95 & 8.86 & 8.14 & 7.06 & 7.84 & 8.53 & 7.94 \\
\hline$m_{\mathrm{RKR}-}$ INR $[\mathrm{kg}]$ & 7.13 & 6.61 & 5.28 & 5.72 & 5.60 & 5.00 & 5.56 \\
\hline$m_{\mathrm{SQT} 2}[\mathrm{~kg}]$ & 2.03 & 2.24 & 2.37 & 2.38 & 2.21 & 2.04 & 2.08 \\
\hline$m_{\mathrm{RBW}}[\mathrm{kg}]$ & 1.75 & 1.65 & 1.80 & 1.72 & 1.24 & 1.35 & 1.26 \\
\hline$m_{\mathrm{RR}-\mathrm{OTR}}[\mathrm{kg}]$ & 1.92 & 1.45 & 1.43 & 1.48 & 1.43 & 1.43 & 1.43 \\
\hline$\Sigma m[\mathrm{~kg}]$ & 31.65 & 30.85 & 29.21 & 28.33 & 27.47 & 27.20 & 27.07 \\
\hline$\Sigma p[€]$ & 56.91 & 56.37 & 58.51 & 61.22 & 66.07 & 68.23 & 73.39 \\
\hline LWC $[€ / k g]$ & - & -0.67 & 0.66 & 1.30 & 2.19 & 2.54 & 3.60 \\
\hline$\delta_{\min }[\mathrm{mm}]$ & 13.72 & 13.16 & 12.82 & 11.91 & 12.84 & 12.02 & 11.62 \\
\hline
\end{tabular}

Optimum F represents the mass optimum and is known from Sect. 4. It offers a weight reduction of $4.58 \mathrm{~kg}$ and $16.48 €$ of extra cost and thus lightweight cost of $3.6 € / \mathrm{kg}$. The B-Pillar of this optimum is not nested.

\section{Conclusion and discussion}

This work presents a methodology to systematically select the most suited parts for the application of TRB in the automotive body. Respecting the computational effort of optimization under consideration of crash load cases a priori selection is presented first. This step enables the proper selection of TRB candidate parts based on formalized engineering rules. Suitable part characteristics as well as the methodology to assess the load inhomogeneity are presented.

A priori preferencing is well suited to reduce the number of design variables to a level where optimization is possible. Especially the inhomogeneity assessment has to be furtherer developed to cope with load cases from different disciplines resulting in field results in different orders of magnitudes like crash and stiffness.
Using metamodels, the optimization of every possible combination of TRB part selections of the remaining part set, the pareto optimal part selection can be found efficiently, revealing mass and cost trade-offs.

Funding Open Access funding enabled and organized by Projekt DEAL.

\section{Declaration}

Conflict of interest On behalf of all authors, the corresponding author states that there is no conflict of interest.

Replication of results The data that support the findings of this study are available from the corresponding author upon reasonable request.

Open Access This article is licensed under a Creative Commons Attribution 4.0 International License, which permits use, sharing, adaptation, distribution and reproduction in any medium or format, as long as you give appropriate credit to the original author(s) and the source, provide a link to the Creative Commons licence, and indicate if changes were made. The images or other third party material in this article are included in the article's Creative Commons licence, unless indicated otherwise in a credit line to the material. If material is not included in the article's Creative Commons licence and your intended use is not permitted by statutory regulation or exceeds the permitted use, you will 
need to obtain permission directly from the copyright holder. To view a copy of this licence, visit http://creativecommons.org/licenses/by/4.0/.

\section{References}

Belyaev M, Burnaev E, Kapushev E, Panov M, Prikhodko P, Vetrov D, Yarotsky D (2016) Gtapprox. Adv Eng Softw 102:29-39. https:// doi.org/10.1016/j.advengsoft.2016.09.001

Biscani F, Izzo D (2020) esa/pagmo2. https://doi.org/10.5281/zenodo. 1045336

CCSA (2016) 2010 toyota yaris finite element model validation detail mesh. https://doi.org/10.13021/G8CC7G, https://media.ccsa.gmu. edu/cache/NCAC-2012-W-005.pdf

Chuang CH, Yang RJ, Li G, Mallela K, Pothuraju P (2008) Multidisciplinary design optimization on vehicle tailor rolled blank design. Struct Multidisc Optim 35:551-560. https://doi.org/10. 1007/s00158-007-0152-0

Deb K, Pratap A, Agarwal S, Meyarivan TAMT (2002) A fast and elitist multiobjective genetic algorithm: Nsga-ii. IEEE Trans Evol Comput 6:182-197

Duan L, Sun G, Cui J, Chen T, Li G (2015) Lightweight design of vehicle structure with tailor rolled blank under crashworthiness. In: 11th World congress on structural and multidisciplinary optimization

Forsberg J, Nilsson L (2005) On polynomial response surfaces and kriging for use in structural optimization of crashworthiness. Struct Multidisc Optim 29:232-243. https://doi.org/10.1007/ s00158-004-0487-8

Harzheim L, Saul M (2004) Optimierung einer karosseriestruktur unter einsatz von blechen mit variabler wandstärke. VDI-Berichte 1846:125-136

Hwang CI, Masud ASM, Paidy SR (1979) Multiple objective decision making. Lecture notes in economics and mathematical systems, vol 164. Springer, Berlin
IIHS (2017) Side impact crashworthiness evaluation - crash test protocol. IIHS, Bengaluru

IIHS (2020) Side impact crashworthiness evaluation 2.0 crash test protocol (version i). IIHS, Bengaluru

Klinke N (2021) Strategien zur optimierung von flexibel gewalzten bauteilen in karosseriestrukturen

Klinke N, Schumacher A (2016) Finding the best thickness run parameterization for optimization of tailor rolled blanks. In: Conference proceedings of 14th German LS-Dyna forum

Klinke N, Schumacher A (2018) Parameterization setup for metamodel based optimizations of tailor rolled blanks. In: Schumacher A, Vietor T, Fiebig S, Bletzinger KU, Maute K (eds) WCSMO 2017: advances in structural and multidisciplinary optimization. Springer, Cham, pp 1833-1850

Klinke N, Schumacher A (2021) Optimization of tailor rolled blanks under consideration of function, formability and cost. Thin-Walled Struct 161:107437

Kodiyalam S, Yang RJ, Gu L, Tho CH (2004) Multidisciplinary design optimization of a vehicle system in a scalable, high performance computing environment. Struct Multidisc Optim 26:256-263. https://doi.org/10.1007/s00158-003-0343-2

Redhe M, Forsberg J, Jansson T, Marklund PO, Nilsson L (2002) Using the response surface methodology and the d-optimality criterion in crashworthiness related problems. Struct Multidisc Optim 24:185-194. https://doi.org/10.1007/s00158-002-0228-9

Stander P, Roux P, Goel T, Eggleston P, Craig P (2015) Ls-opt user's manuel a design optimization and probabilistic analysis tool for the engineering analyst.

Publisher's Note Springer Nature remains neutral with regard to jurisdictional claims in published maps and institutional affiliations. 